\title{
THE CURVED-CRYSTAL SPECTROMETER AT THE UNIVERSITY OF MICHIGAN
}

\author{
J. J. REIDY and M. L. WIEDENBECK
}

University of Michigan, Ann Arbor, Michigan

Received 3 October 1964

A precision curved-crystal spectrometer, following the design of Seppi, Henrickson, Boehm and DuMond, has been built and put into operation. The use of a tantalum collimator and germanium crystals has greatly extended the useful range of this instrument.

\section{Introduction}

Curved-crystal diffraction spectrometers make use of the focussing Bragg-reflection property of a curvedcrystal and have been described by many authors with a good list of references being available in a review article by DuMond ${ }^{1}$ ). The most popular spectrometer design sometimes called the monochromator arrangement is one where the line source is situated on the focal circle through the neutral axis of the curvedcrystal. The gamma-rays scattered at the Bragg angle pass through a collimator and are detected by a $\mathrm{NaI}(\mathrm{Tl})$ spectrometer. The monochromator arrangement can be of several types. One type uses a stationary collimator and detector system with the source and crystal coupled in such a manner that the Bragg condition is always maintained. An example of this type is the Caltech Mark I instrument ${ }^{1}$ ). However, the more versatile type of monochromator is one with a stationary source and the crystal and collimator coupled in the proper manner.

Originally the curved-crystal used in a monochromator was quartz. Sources of high activity were required, often of the order of tens or hundreds of curies. As a result only the decay of reactor produced isotopes could be studied. Even then thermal neutron absorption cross-sections of the order of tens of barn and a high thermal neutron flux (of the order of $10^{14}$ neutrons $/ \mathrm{sec} \cdot \mathrm{cm}^{2}$ ) were generally a prerequisite. In addition, since quartz is composed of low atomic number elements, the efficiency for scattering high energy gamma radiation is inherently small. This further limited the monochromator to the precision measurement of gamma-rays with energies generally below $1 \mathrm{MeV}$.

However, recent advances in the techniques of growing large single crystals of higher atomic number materials (germanium, in particular) have greatly increased the possible uses of the curved-crystal monochromator. A description of a germanium curvedcrystal monochromator has already been reported by
Results are presented concerning the relative properties of four crystals; quartz (310), quartz (112), germanium (400) and germanium $(0 \overline{2} 2)$. Measurements of the energies of the gammarays following the decay of $\mathrm{Au}^{198}$ and $\mathrm{Au}{ }^{199}$ are also presented.

Seppi et al. $^{2}$ ). From their study it was evident that compared with quartz one not only obtained higher reflection efficiency at short wavelengths with germanium but there was also a considerable improvement in resolution. It now appeared that precision measurements of gamma-ray energies in the $2-3 \mathrm{MeV}$ region might be possible with such an instrument. Certainly more complicated decay schemes could be studied, perhaps even in isotopes produced in medium flux reactors from parent isotopes with thermal neutron absorption cross sections of the order of a barn. The improvement in resolution also implies an improvement in the determination of the line position (and, hence, the energy of the transition) and perhaps increases the feasibility of determining the isomeric shift which is expected for certain X-ray transitions. Further, the improvement in resolution would be an invaluable aid in the study of the natural line width of X-rays.

These and other considerations indicated that a great deal of valuable data could be obtained with a curvedcrystal monochromator. Such an instrument using a stationary source has been built at the University of Michigan. A description of the U-M instrument and some studies of its characteristics are presented in this paper. Four diffraction crystals, two quartz and two germanium, all with different orientations, are used with this instrument at the present time, and data concerning the properties of these crystals are presented.

\section{Monochromator design}

\subsection{CRystal pivot and DETECTOR CARRIAGE}

The U-M monochromator is a duplicate of the Caltech germanium 2-meter bent-crystal monochromator described by Seppi et al. ${ }^{2}$ ) except for some minor variations. The crystal pivot unit consists of the curved diffraction crystal, the pivot bearing and the precision sine-screw mechanism. The precision lead screw moves the lever arm which rotates the crystal about the axis provided by the pivot bearing. The sine of the Bragg 
angle (and, thus the wavelength) can then be read directly on a dial attached to the screw. The dial is calibrated in screw divisions (s.d.) and the design is such that one s.d. is approximately equal to one X-unit when the diffraction is from the (310) planes of quartz. Equivalently, one screw division is approximately equal to ninety second of arc. Although there is a calibration cam which can be used to correct for small nonlinearities in the pitch of the lead screw, there is no indication that this correction is necessary in our instrument. A total of 47 measurements of gamma-ray energies were made using all four crystals. These measurements were obtained for various orders of reflection and in none of these measurements were there any inconsistencies which could be attributed to non-linearity in the pitch of the lead screw. In addition, the monochromator error $E_{\mathrm{m}}$ discussed by Seppi et al. ${ }^{2}$ ) was determined to be 0.0033 s.d. for our instrument. The value of $E_{\mathrm{m}}$ obtained by Seppi et al. after correcting for non-linearities in the screw of their instrument was 0.003 s.d. Precision stepwise motion of $0.04,0.02,0.01$, or 0.005 s.d. per step is obtained by using a direct current Slo-Syn stepping motor which is directly coupled to the lead screw. Rapid motion from one setting to another is obtained by applying alternating current to this motor in the proper manner. The detector-carriage unit contains the shielded detector and collimator. These components are carried on a platform which is constrained to move in such a way that

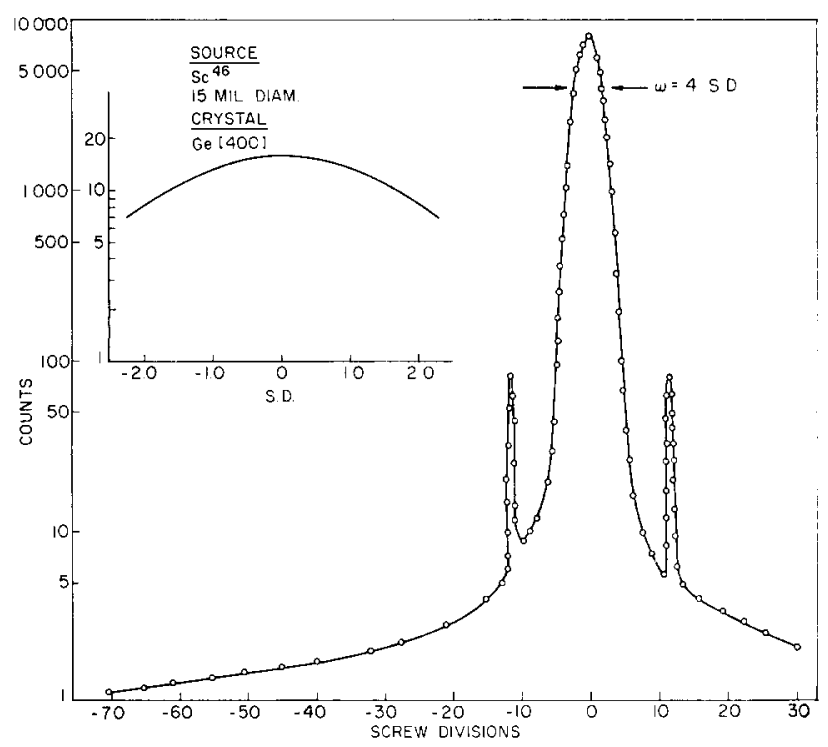

Fig. 1. Gamma-ray intensity vs crystal position. The sharp peaks correspond to the first order reflection of the $889 \mathrm{keV}$ gamma-ray. The inset shows an expanded portion of the central peak which is due to the undiffracted gamma-ray beam. the Bragg condition is satisfied. The motion of the platform is electrically linked to that of the crystal pivot. The collimator consists of 49 tantalum sheets each being $3 \times 18 \times 0.020$ in. The sheets are spaced with 0.0625 in. between centers at the entrance. The measured transmission of the collimator is 50 per cent. The angular resolution of the collimator is demonstrated in fig. 1. The peaks correspond to the first order reflection from the (400) planes of germanium of the 889 $\mathrm{keV}$ gamma-ray in the decay of $\mathrm{Sc}^{46}$. The full width at half maximum (fwhm) of the undiffracted beam is 4.0 s.d. or nearly 6 minutes of arc. At higher energies one would expect this to be slightly larger. The inset in fig. 1 shows the shape of the curve due to the undiffracted beam near the 0.0 s.d. region. The curve does not peak but is actually flat on top with a width of 0.4 s.d.*

\subsection{DifFRACTION CRYSTALS AND CRYSTAL HOLDERS}

The Bragg reflection is provided by a thin optically ground crystal lamina clamped between two stainless steel blocks which have been profiled to a two-meter radius of curvature. The blocks are the same type as described by Seppi et al. ${ }^{2}$ ).

At the present time four crystals are available. These are oriented so the quartz (310) or (112) planes or the germanium (400) or $(0 \overline{2} 2)$ planes are used for reflection. The crystals are square lamina each $7.5 \mathrm{~cm}$ by $7.5 \mathrm{~cm} \times 2 \mathrm{~mm}$ thick except the (400) germanium which is $6.3 \mathrm{~cm}$ by $6.3 \mathrm{~cm}$. Before bending, the dislocation density of each crystal is less than 5000 pits $/ \mathrm{cm}^{2}$. The lamina are optically polished to a flatness of about 1 fringe of sodium light.

The $(0 \overline{2} 2)$ germanium was originally intended to be used with the (422) planes as the reflecting planes and it was so oriented. However, after bending this lamina, the results obtained with the (422) planes were not encouraging, so it was decided to abandon this particular orientation. By rotating the crystal lamina through $90^{\circ}$ and then bending it, reflections due to another set of planes were discovered. From a determination of the crystal plane spacing (approx. $2.000 \AA$ ) and the fact that these planes must be perpendicular to the (422) planes, the reflection is attributed to the $(0 \overline{2} 2)$ planes. The $(400)$ germanium is smaller than

* A study was made to determine the advisability of using two additional collimators. A pre-collimator of vertical tantalum sheets was placed in the beam near the source. A post-collimator consisting of horizontal tantalum sheets was inserted between the collimator and $\mathrm{NaI}(\mathrm{Tl})$ detector. The overall effect with either or both these additional collimators was not deemed large enough to warrant their use. 


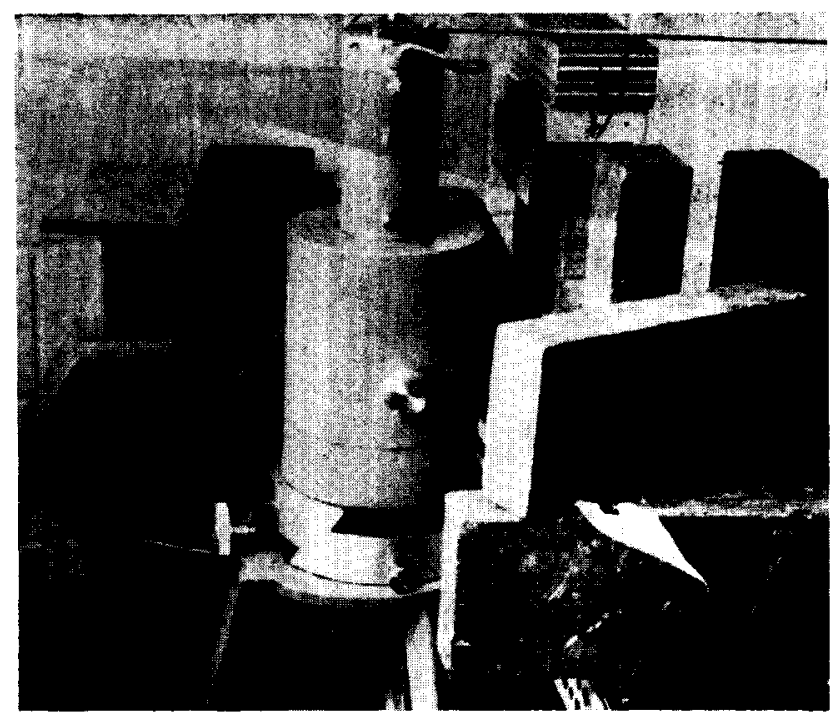

Fig. 2. Photograph of the source holder.

optimum size; but, at the time it was ordered, there was not a larger size available.

Of the four crystals, only the complete aperture of the (310) quartz is useful. Proper shielding must be used in order to obtain optimum results with the other crystals.

\subsection{SOURCE HOLDER ARRANGEMENT}

A photograph of the source holder arrangement consisting of a table and the source holder unit is shown in fig. 2. The table is constructed of welded square steel tubing, provided with three leveling screws. The source holder unit, shown schematically in fig. 3a, consists of a base, the source shield and source holder and a lead pig. The base is bolted to the table but is so constructed that the upper portion is movable in perpendicular directions in the horizontal plane by means of two dovetail carriages. The lead source shield has a diameter of 6.25 in. and contains a small slotted brass tube to accept and hold in alignment, the source bearing capsule. The upper part of the shield is machined to accept the lead pig in which the source capsule is carried and stored. The source capsule can be inserted into its operating position by lowering it from the carrying pig through a hole in the source shield into the aligned holder.

\section{Source technique, preparation and handling}

A considerable effort has been put into the question of source handling with the following goals in mind: 1. safety and ease of handling sources up to 20 curies or more; 2 . accuracy and speed in alignment of source; 3. ease of preparation of source material.
It was decided that the sources should be prepackaged before irradiation in such a manner so that no extensive preparatory work need be done on the hot source before inserting it into the spectrometer. Initially, wire or powder sources were used by sealing the material in a straight quartz tube of appropriate inner diameter ( 2 to 5 mils) and approximately 32 mils outer diameter with a length of one inch. The quartz source tube was then sealed into an aluminum tube $\frac{1}{8}$ " outer diameter, $1 \frac{1}{4}$ " long which was individually drilled from both ends to assure a tight fit and accurate centering of the quartz tube. Capsules of 0.25 in. diameter may also be used if the appropriate aligning holder is inserted in the source shield. This double sealed package was then irradiated either in the Oak
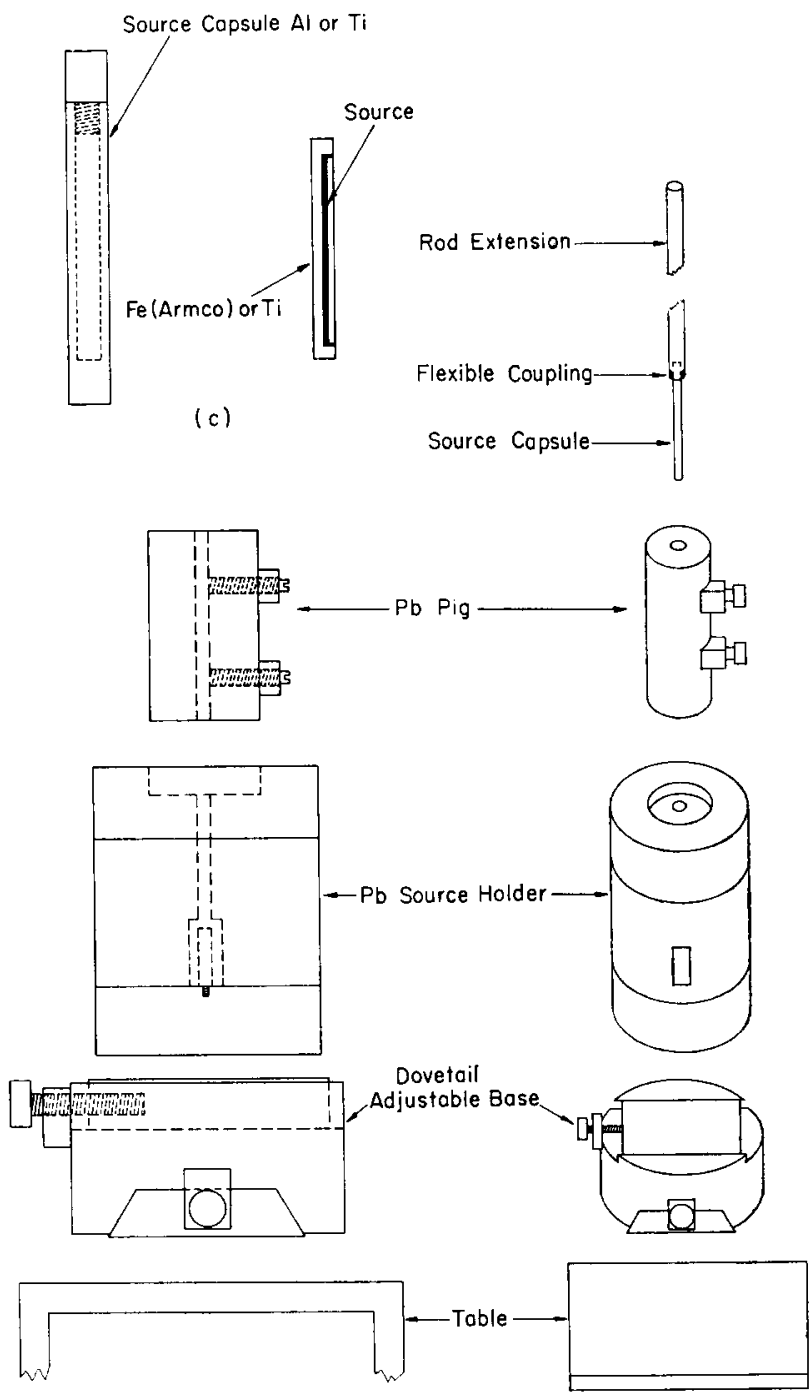

(a)

(b)

Fig. 3. Schematic of the source holder arrangement. 
Ridge Research Reactor, or, more commonly, in the U. of M. Ford Reactor. (The Ford Reactor is a $2 \mathrm{MW}$ swimming pool type with a flux $5 \times 10^{12} \mathrm{n} / \mathrm{cm}^{2}$. sec.)

In recent months we have found it more convenient to use flat (ribbon) sources with the same system because of greater ease of preparation and also to obtain greater quantities of source material without effectively increasing the source width as seen by the crystal. In this system the quartz source tube is replaced by a pure iron or titanium half-cylinder one inch long and diameter $\frac{1}{16}$ inch. See fig. 3c. The flat part is covered with a mixture of the source material in epoxy and machined flat to the thickness required (usually 2 to 5 mils). This source is then inserted into the $\frac{1}{8}$ inch capsule for irradiation.

After the source has been irradiated the aluminum cylinder is secured in the lead carrying pig three inches in diameter and 6 inches high. (This operation is carried out under water in the reactor pool.) The lead pig containing the source is then transported (with appropriate additional shielding) to the spectrometer and a rod is attached to the source capsule by means of a flexible, push type O-ring coupling. See fig. $3 b$. The source pig is then put in position above an accurately aligned tube in the spectrometer source shield whereupon the source is simply pushed through the pig into position. Normally, no further alignment is required with a cylindrical source. After the source has been used it is withdrawn into the carrying pig and stored until it is again needed.

Since it is required that the crystal should "look" at the thin edge of the ribbon for best resolution, additional, careful alignment of the flat source is necessary. This is accomplished by attaching a 50 tooth worm gear arrangement to the brass rod so that the source may be rotated through small angles. This arrangement appears at the top of fig. 2. The monochromator is then set to detect a given gamma-ray energy occurring in the source and the source is rotated until the maximum counting rate is achieved. Sometimes slight changes must be made in the monochromator setting since rotating the source can shift it tangentially to the focal circle, particularly if the source is not symmetric about the axis of the source holder. As a rule the sources are so well made that this change is unnecessary. With a little practice these flat sources can be aligned in a matter of minutes. On the average it takes less than thirty minutes from the time the source is removed from the reactor core until it is aligned in the monochromator. In special cases this time interval can be made less than fifteen minutes.

\section{Monochromator energy calibration}

No attempt was made to calibrate the monochromator in terms of absolute wavelength; instead, a calibration setting was determined for each crystal whereby the setting in screw divisions is converted directly to energy in $\mathrm{keV}$. The transition which appears to be well suited for this role is the $411.8 \mathrm{keV}$ transition in $\mathrm{Hg}^{198}$.

From the recent work of Murray et al. ${ }^{3}$ ) concerning the $411.8 \mathrm{keV} \mathrm{Hg}^{198}$ gamma-ray, it was felt that one can assign the value $411.800 \mathrm{keV}$ to it and this value will be consistent with all the known precision energy determinations for this transition. Large amounts of the parent isotope $\mathrm{Au}^{198}$ are easy to obtain and the half-live is long enough so many measurements can be obtained. For these reasons in the calibration of the $\mathrm{U}-\mathrm{M}$ monochromator the value $411.800 \mathrm{keV}$ has been used for the intense $\mathrm{Hg}^{198}$ gamma-ray and a series of measurements have been performed to determine the screw division settings corresponding to the various orders of reflection of this gamma-ray for each crystal. If it becomes evident that the energy value of 411.800 $\mathrm{keV}$ for the $\mathrm{Hg}^{198}$ transition needs revising, it is an easy matter to change the calibration of the energies of the measured gamma-rays. Also, data obtained with another instrument may be compared with the data obtained with the $\mathrm{U}-\mathrm{M}$ monochromator if this $\mathrm{Hg}^{198}$ gamma-ray has been measured with the former instrument and suitably normalized to this $411.800 \mathrm{keV}$ value for the energy.

Gold sources ranging in size from a one mil diameter wire to a foil $0.005 \times 0.125$ in. with a maximum activity of three curies were used in the measurements. The calibration constants and their associated uncertainties are presented in table 1 .

The monochromator error $E_{\mathrm{m}}$, which has been discussed by Seppi et $\mathrm{al}^{2}{ }^{2}$ ), was determined from the series

TABLE 1

Position of the first order diffraction peak for $411.800 \mathrm{keV}$ gamma-ray and the resultant calibration constant for each crystal. The energy of a gamma-ray is obtained by dividing the calibration constant by the position (in screw divisions) of the diffracted peak corresponding to first order reflection of the gamma-ray from the respective crystal.

\begin{tabular}{l|c|r} 
Crystal & $\begin{array}{c}\text { Position of line corresponding } \\
\text { to first order reflection of the } \\
411.800 \mathrm{keV} \text { gamma-ray in } \\
\mathrm{Hg}^{198} \text { (s.d.) }\end{array}$ & $\begin{array}{c}\text { Calibration } \\
\text { constant } \\
\text { (keV-s.d.) }\end{array}$ \\
\hline & $30.0387 \pm 0.0012$ & $12369.94 \pm 0.50$ \\
(310) Quartz & $19.6735 \pm 0.0010$ & $8101.55 \pm 0.41$ \\
(112) Quartz & $25.0647 \pm 0.0013$ & $10321.64 \pm 0.52$ \\
$(400) \mathrm{Ge}$ & $17.7233 \pm 0.0004$ & $7298.45 \pm 0.18$ \\
\hline$(022) \mathrm{Ge}$ & & \\
\hline
\end{tabular}


of calibration runs for all the crystals in addition to data obtained for the higher energy lines in the decay of $\mathrm{Au}^{198}$. In all, a total of 47 measurements were taken. These include measurements with the $(0 \overline{2} 2)$ germanium of the line position of the $411.800 \mathrm{keV}$ gamma-ray through the seventh order reflection, of the $676 \mathrm{keV}$ gamma-ray through the third order reflection and of the $1088 \mathrm{keV}$ gamma-ray in second order reflection. With the (400) germanium and the (112) quartz measurements of the $411.800 \mathrm{keV}$ gamma-ray through third order reflection were obtained. Only measurements corresponding to first order reflection of this gamma-ray were obtained with the (310) quartz. The value for $E_{\mathrm{m}}$ was found to be $0.0033 \mathrm{~s}$.d. No error due only to temperature variations has been included since the temperature of the room is kept constant within $1.5^{\circ} \mathrm{C}$ during the accumulation of data. Instead it is felt that a first order correction for an error due to temperature effects will be contained in $E_{\mathrm{m}}$.

\section{Measurement techniques}

\subsection{STRIP CHART RECORDER}

A study of the decay of a given isotope generally begins with a scan of a large gamma-ray energy interval using a strip chart recorder. The output of the $\mathrm{NaI}(\mathrm{Tl})$ detector is connected to ground through a ten turn helipot. The helipot is electrically coupled to the motion of the precision lead screw. One turn of the helipot corresponds to 40 screw divisions with the helipot reading zero at zero screw division. Consider now only the detection of the reflected gamma-ray. The voltage output pulse from the $\mathrm{NaI}(\mathrm{Tl})$ detector is proportional to the inverse of the gamma-ray wavelength and, therefore, to the inverse of the number of screw divisions. Since the setting of the helipot is proportional to the number of screw divisions, the voltage output of the helipot must necessarily be a constant, independent of the energy of the diffracted gamma-ray. Furthermore, for the second order reflection the output voltage of the helipot will be twice as great as for first order reflection, three times as large for third order reflection, etc. By putting the output of the helipot into an amplifier-discriminator, one is now able to record all the gamma-rays which undergo a reflection of a particular order. This may be done over a large gamma-ray energy range with a greatly increased peak-to-background ratio compared with integral counting over a similar range. The output of the discriminator is put into a count rate meter which in turn drives a strip chart recorder. In this manner, a spectrum from several hundred $\mathrm{keV}$ to a few tens of $\mathrm{keV}$ may be scanned automatically. These scans are never used to determine precise gamma-ray energies, but are used to determine the possible regions for more careful study.

\subsection{Precision measurements of gamma-Ray enERgies}

For precision energy determinations, the helipot is seldom used. Instead, the output of the $\mathrm{NaI}(\mathrm{Tl})$ is put into the amplifier-discriminator. The output of the discriminator is counted by a scaler and recorded on a Sodeco paper tape print out register. The control of the stepping motor is generally set so data will be recorded at a minimum of twenty points as the monochromator traverses the peak due to the reflected gamma-ray. Data at more than twenty points are always taken since the background on either side of the peak must always be determined.

This procedure is followed for reflection from both sides of the curved-crystal planes with the monochromator always proceeding in the same direction rotationwise. The two sets of data are then corrected for source decay, if necessary, and plotted. The shape of the background is determined and a point-by-point subtraction of the background from the data points is performed. The resulting data are normalized and plotted. The two plots are superimposed and a visual determination of the possible region of overlap is made. Each data point is assigned its proper statistical error. The maximum and minimum possible values of the wavelength of the gamma-ray (or integral multiple thereof, if the data are obtained for order of reflection greater than one) are obtained by requiring that, in the worse case, only a small region (determined by two or three adjacent points with their associated errors) of one plot need overlap a similar region of the other plot. The wavelength of the gamma-ray (or integral multiple thereof) in screw division units with an associated uncertainty is thus determined. For convenience this uncertainty is called the statistical error $E_{\mathrm{s}}$ although it is certainly larger than the error in the position of the line resulting from only statistical counting errors. The uncertainty finally attached to the measurement of a given line is $E$ where $E^{2}=E_{\mathrm{s}}^{2}+E_{\mathrm{m}}^{2} . E_{\mathrm{m}}$ is the monochromator error discussed in the previous section.

Data obtained at higher order reflections are now converted to values corresponding to first order reflection and the average value of the wavelength (in s.d.) is determined. The energy of the gamma-ray is calculated by dividing the appropriate calibration constant in table 1 by this average value of the wavelength. The uncertainty $E$ in each measurement is also converted to the appropriate value corresponding to first order reflection. The uncertainties are added quadratically to obtain the uncertainty in the average value of 


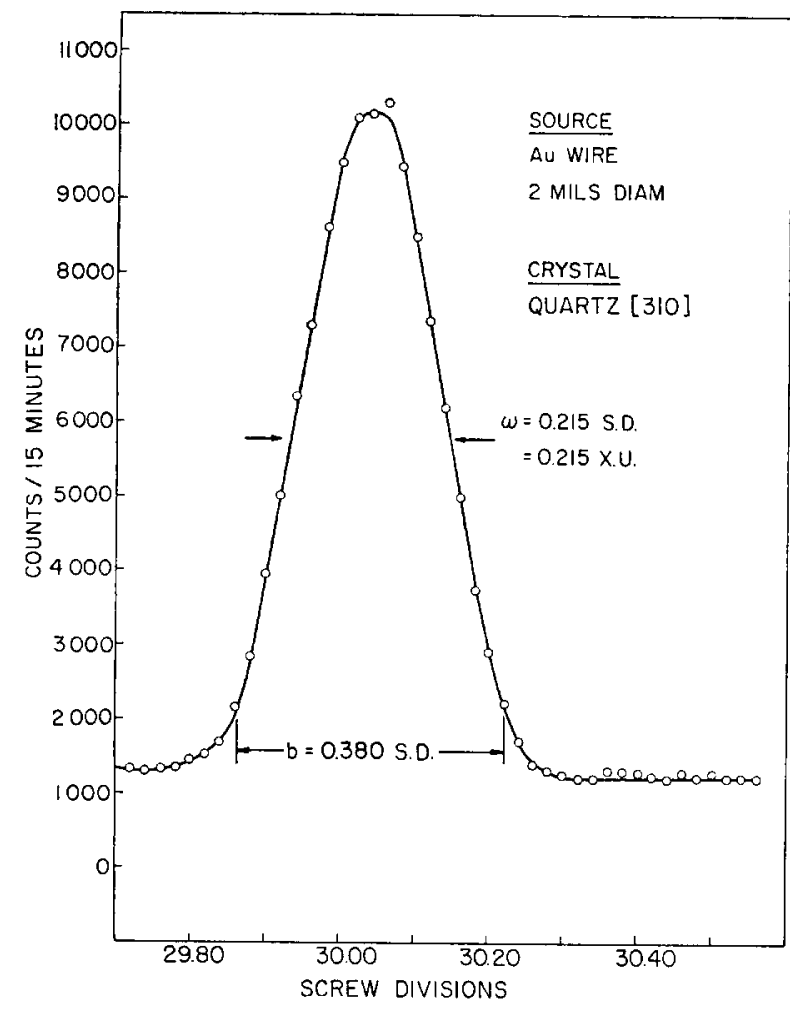

Fig. 4. The $411.8 \mathrm{keV}$ Au ${ }^{198}$ gamma-ray line observed in first order reflection. The full width at half-maximum intensity (fwhm) is designated $\omega$ and the width at ten percent maximum intensity is designated $b$.

the wavelength. The fractional uncertainty in the energy is found by adding quadratically the fractional uncertainties in the average wavelength of the gammaray and the calibration constant.

\section{Properties of the various curved-crystals}

\subsection{THE (310) AND (112) QUARTZ}

Of the four crystals, only the (310) quartz is usable over its whole area. The exposed area of a crystal in the clamping block is approximately $20 \mathrm{~cm}^{2}$. The (112) quartz crystal has undergone twinning and must be shielded so its maximum usable area is about $9 \mathrm{~cm}^{2}$. Fig. 4 shows the results obtained with the (310) quartz for the first order reflection of the $411.800 \mathrm{keV}$ transition. The source is a 0.002 in. diameter wire of about 10 mCur. The quantity $\omega$ is the full width at half maximum ( $\mathrm{fwhm}$ ) and the base width, $b$, is defined as the width of the peak at $10 \%$ of the peak intensity. Fig. 5 shows the results obtained with the (112) quartz for the second order reflection of the $411.800 \mathrm{keV}$ gamma-ray with the source also being a $0.002 \mathrm{in.}$ diameter wire. Comparing figs. 4 and 5 , it is interesting

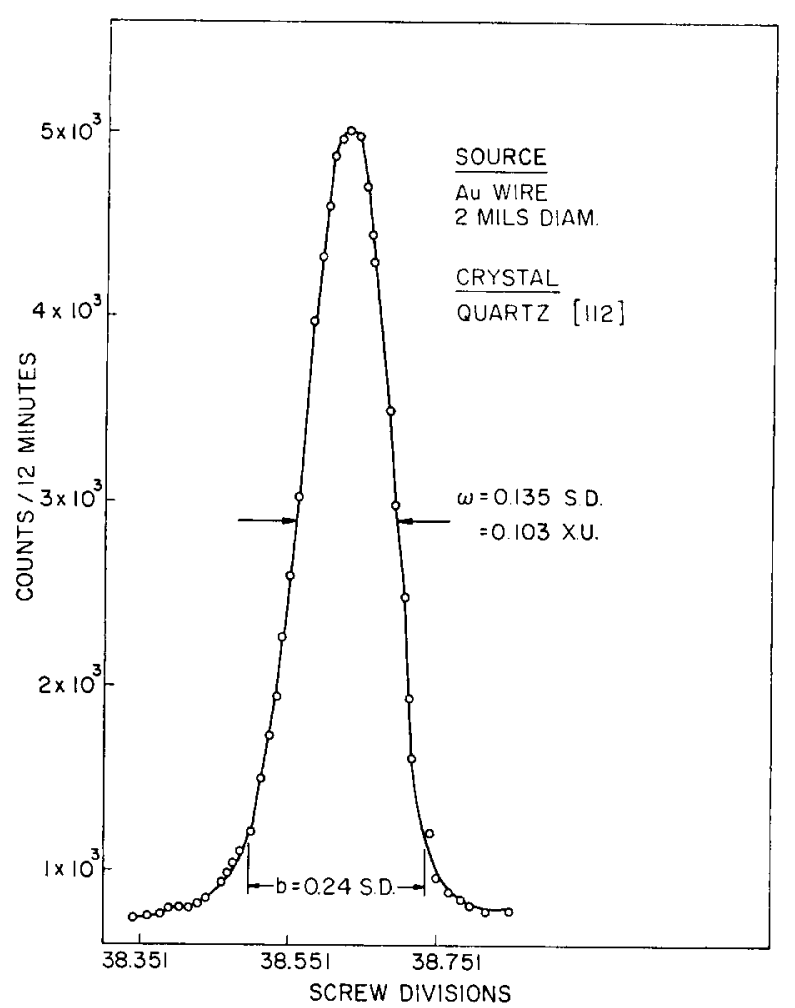

Fig. 5. The $411.8 \mathrm{keV}$ gamma-ray line observed in second order reflection.

to note that although the fwhm in s.d. units is appreciably smaller for the line obtained with the (112) quartz, the fwhm in X.units is roughly the same for the same order of reflection from both of these crystals. For first order reflection of the $411.800 \mathrm{keV}$ transition using the (112) quartz, the fwhm is 0.204 X.u.

\subsection{The (400) AND $(0 \overline{2} 2)$ GERMANiUm CRYstals}

Both germanium crystals have maximum useful areas of about $5 \mathrm{~cm}^{2}$. Fig. 6 shows a result obtained with the (400) germanium for the first order reflection of the $411.800 \mathrm{keV}$ gamma-ray. The source was a 0.001 in. diameter gold wire of about $15 \mathrm{mCur}$. Using a 0.002 in. diameter source the fwhm of the peak corresponding to the same gamma-ray is $0.080 \mathrm{X}$.u. It is interesting to note that this latter value is only half as large as that found by Seppi et al. ${ }^{2}$ ) for a source of the same dimensions. Fig. 7 shows the results obtained with wire sources of various diameters. In all cases the maximum crystal area was used which would give the maximum peak intensity without loss of resolution. From this figure one finds that, using the (400) germanium and a wire source, the minimum fwhm for a gamma-ray line is given by 


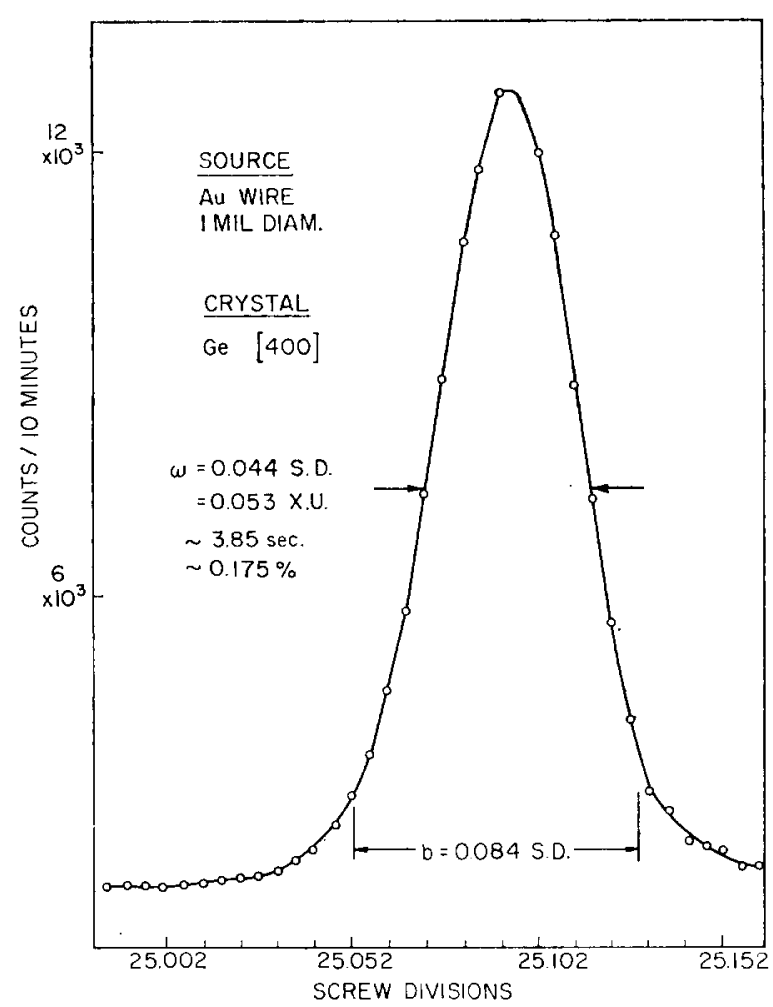

Fig. 6. The $411.8 \mathrm{keV}$ gamma-ray line observed in first order reflection. The fwhm is $0.78 \mathrm{keV}$.

$$
\Delta \lambda=(0.032+0.025 d) \frac{1}{n} \quad \text { X.u. }
$$

or

$$
\Delta E=(2.6+2.0 d) \times 10^{-6} \frac{E^{2}}{n} \quad \mathrm{keV},
$$

where $d$ is the source diameter in mils, $n$ the order of reflection and $E$ the energy of the gamma-ray in $\mathrm{keV}$. The constant term $0.032 \mathrm{X} . \mathrm{u}$. is of interest since it is,

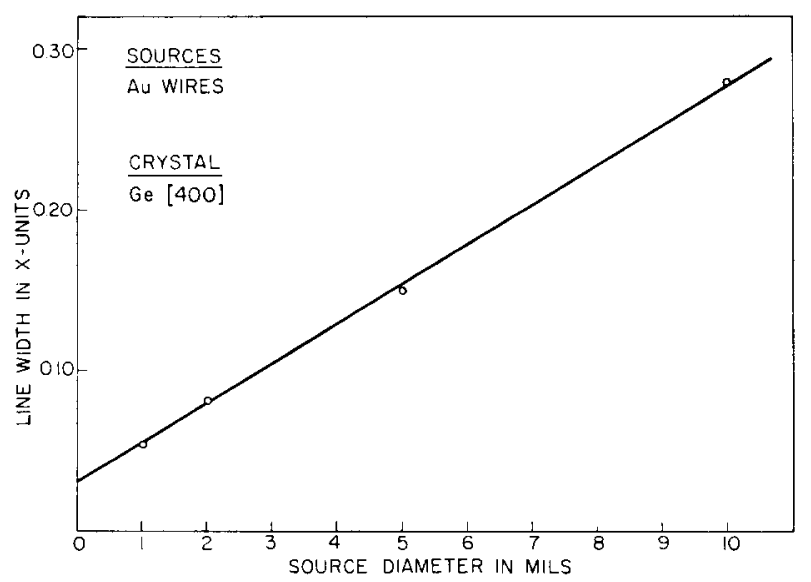

Fig. 7. Line width (fwhm) vs source diameter for the germanium (400) crystal.

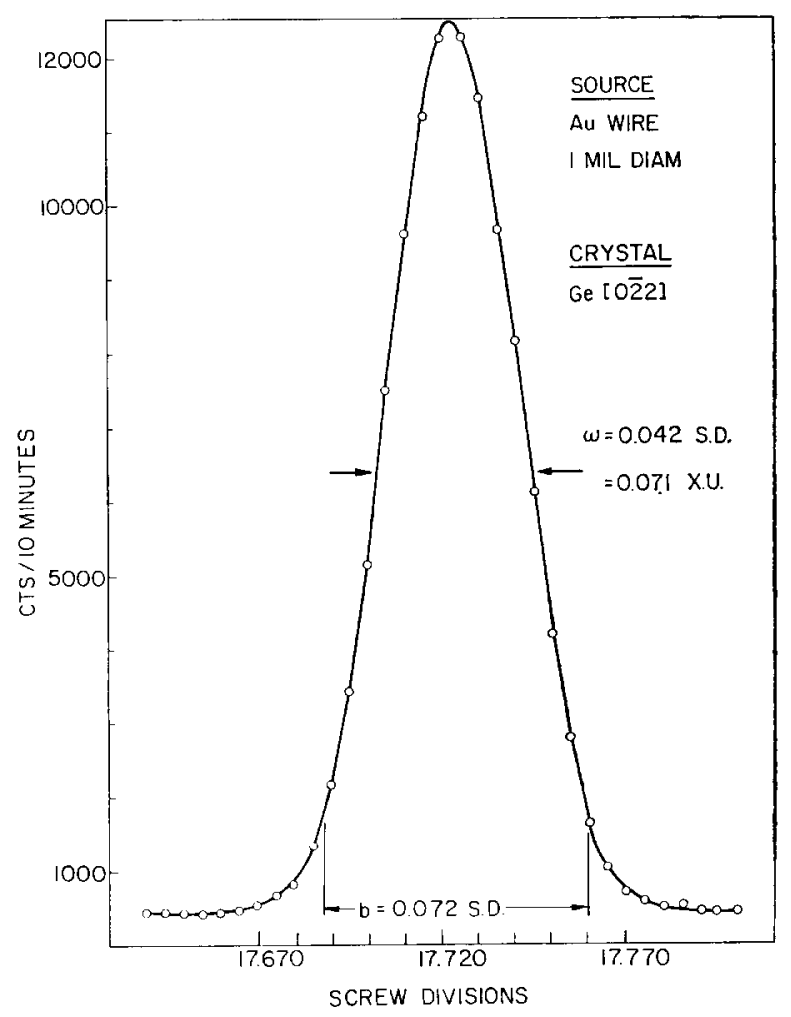

Fig. 8. The $411.8 \mathrm{keV}$ gamma-ray line observed in first order reflection. The fwhm is $0.96 \mathrm{keV}$.

at the least, an upper limit for the value of the line width caused by the crystalline structure of the curved crystal.

As was mentioned earlier the $(0 \overline{2} 2)$ germanium crystal was obtained by rotating a (422) crystal through $90^{\circ}$. The (422) crystal was first bent but the resulting peaks were quite broad with a typical fwhm being 0.50 s.d. or about $0.50 \mathrm{X} . \mathrm{u}$. The corresponding resolution was $4 \times 10^{-5} E$ where $E$ is the gamma-ray energy in $\mathrm{keV}$. This value of the resolution is nearly twice as large as reported by Lind and Henning ${ }^{4}$ ) for the germanium (422) planes and about six times as large as that obtained with our (400) germanium crystal. The peaks were actually flat on top with the flat region being nearly 0.3 s.d. The peak shapes remained unchanged even when widely separated area increments were used for the reflection. This indicates that the peak shapes are a result of a front-to-back change in the crystal structure. One explanation may be that these particular crystal planes can "slip" rather easily with respect to each other enabling the orientation or spacing of these planes to change appreciably when the crystal is bent. However, the $(0 \overline{2} 2)$ orientation gives a very satisfactory set of planes. There are two surface regions which can be used on the $(0 \overline{2} 2)$ crystal with a total maximum 


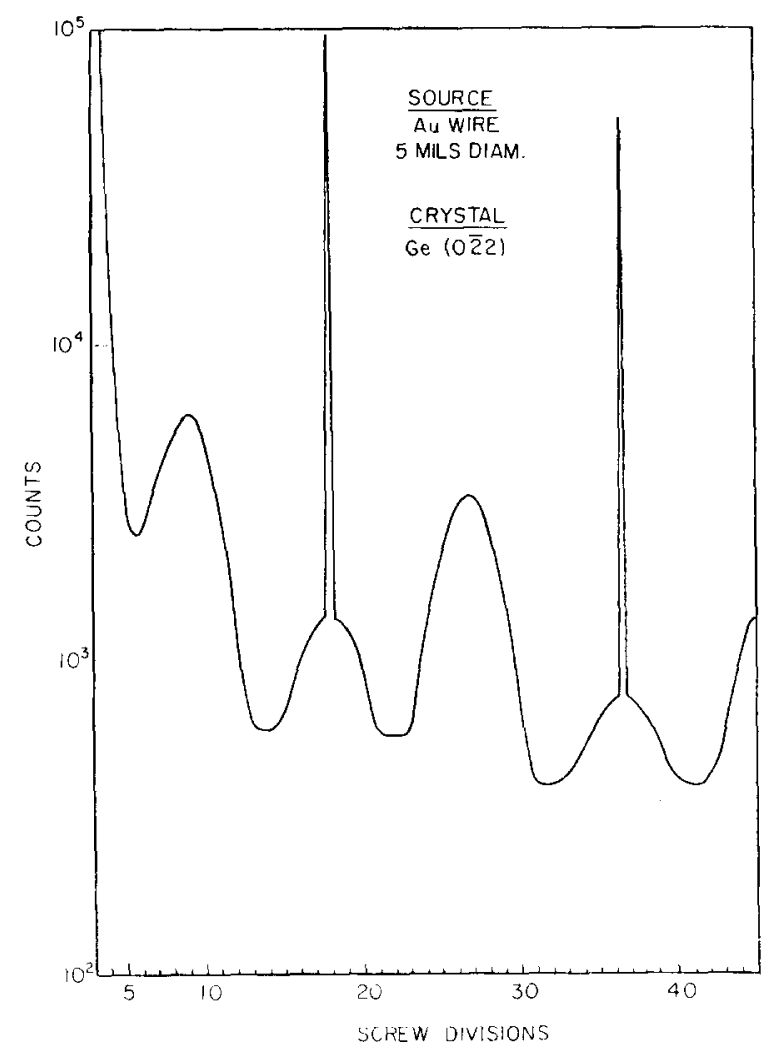

Fig. 9. The $411.8 \mathrm{keV}$ gamma-ray intensity vs crystal position using the germanium $(0 \overline{2} 2)$ planes. The region below 5 s.d. corresponds to the detection of the undiffracted beam. A discussion of the various peaks is given in the text.

useful area of about $5 \mathrm{~cm}^{2}$. These two regions appear to be more uniform than the region in the (400) crystal since the fwhm of a peak changes much less rapidly as one increases the crystal area of the $(0 \overline{2} 2)$ as compared with the (400). Fig. 8 shows a result obtained with the $(0 \overline{2} 2)$ planes of germanium for the first order reflection of the $411.8 \mathrm{keV}$ gamma-ray. The source is a $0.001 \mathrm{in}$. diameter wire of about $7 \mathrm{mCur}$. Wire sources with larger diameters were also studied. From these investigations it was determined that the minimal fwhm of a gammaray line reflected from the $(0 \overline{2} 2)$ planes is given by

$$
\Delta \lambda=(0.036+0.035 d) \frac{1}{n} \quad \text { X.u. }
$$

or

$$
\Delta E=(2.9+2.8 d) \times 10^{-6} \frac{E^{2}}{n} \mathrm{keV} .
$$

The constant term 0.036 X.u. is roughly the same as that obtained in the study of the (400) planes.

Since no known study exists of the curved $(0 \overline{2} 2)$ planes, it seemed advisable to check and see if other planes could also have the proper orientation to give reflections. The discriminator was adjusted so only the detection of the $411.8 \mathrm{keV}$ gamma-ray in the $\mathrm{NaI}(\mathrm{Tl})$ would be observed and a scan was taken of the region from 3 to $100 \mathrm{s.d}$. The data from a portion of this region are shown in fig. 9 . The sharp peaks represent the first and second order reflection of the $411.8 \mathrm{keV}$ gamma-ray from the $(0 \overline{2} 2)$ planes. In addition, it is evident that some property of the crystal is also causing equally spaced broad peaks. However, the peaks corresponding to reflection from the $(0 \overline{2} 2)$ planes are roughly ten times narrower than the broad peaks; so, in practice, no confusion will result from the latter. Some surprising results are obtained from the data which is partially shown in fig. 9. The area under the broad peak at roughly 9 s.d. is equal to the area under the sharp peak corresponding to the first order reflection from the $(0 \overline{2} 2)$ planes ( 17.7 s.d.); the area under the broad peak at about 27 s.d. is equal to the area under the sharp peak corresponding to the second order reflection from the $(0 \overline{2} 2)$ planes $(35.4$ s.d.) and the area under the broad peak at about 45 s.d. is equal to the area under the sharp peak corresponding to the third order reflection from the $(0 \overline{2} 2)$ planes $(52.7$ s.d.). In other words, the total radiation intensity reflected by the crystal into the region corresponding to the broad peak at 9.0 s.d. is equal to the total radiation intensity reflected in first order by the $(0 \overline{2} 2)$ planes, likewise for the peak at 27.0 s.d. and the second order reflection from the $(0 \overline{2} 2)$ planes, etc. It appears that the crystal properties are such that the reflection conditions from the odd indexed $(0 \overline{1} 1)$ planes are satisfied for a relatively large crystal rotation whereas the reflection conditions from the even indexed $(0 \overline{1} 1)$ planes are only satisfied for very small crystal rotations. This investigation has not been extended to the other crystals at

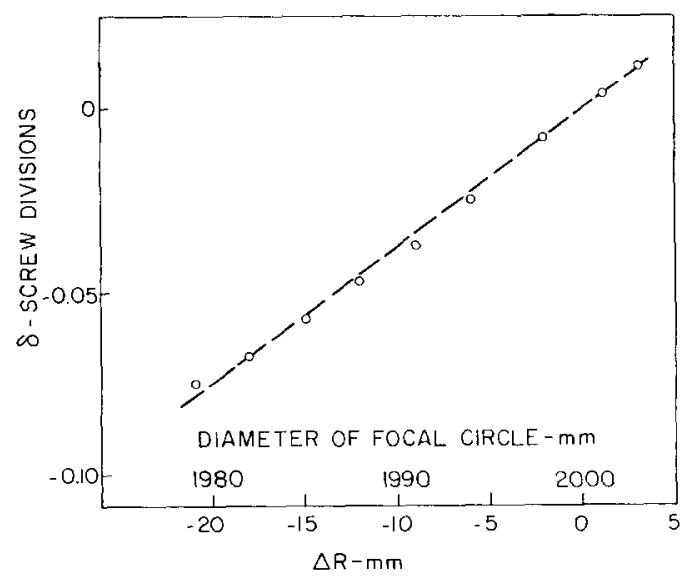

Fig. 10. The change in the diffracted peak position is vs the change in source position $\Delta R$. The diameter of the focal circle is $(2000+\Delta R) \mathrm{mm}$. 


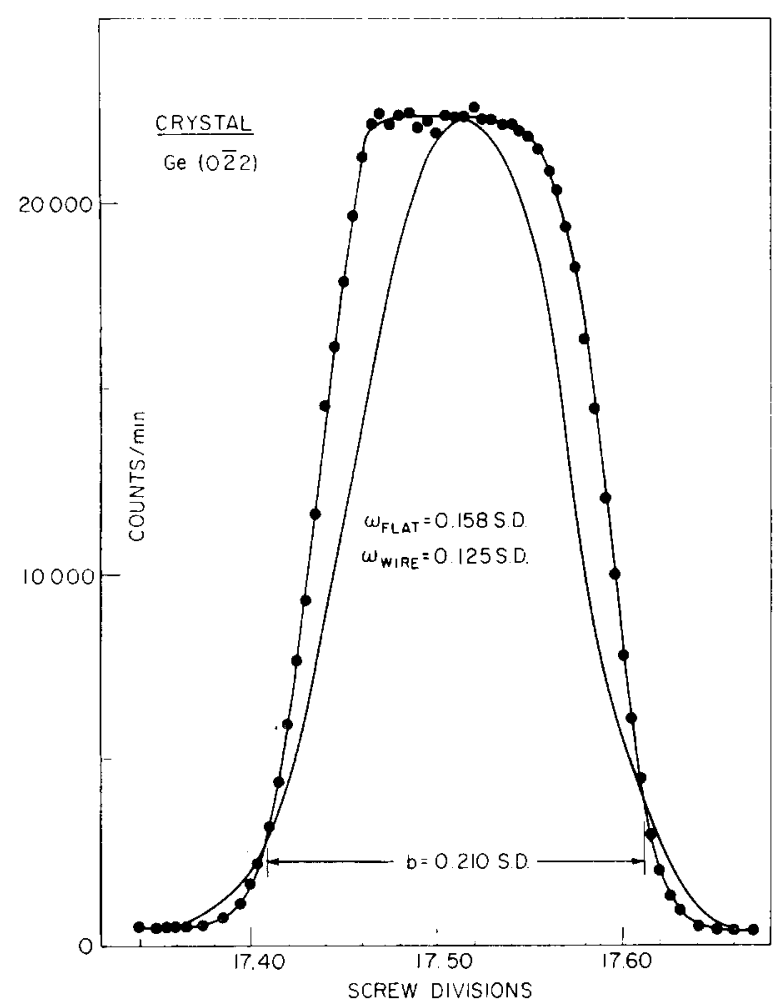

Fig. 11. Relative peak shapes for a five mil diameter wire source and a five mil thick foil source. The two curves have been normalized so each has the same maximum intensity.

this time but it would be interesting to see if a similar effect is noted for them.

An extensive investigation of the feasibility of using flat sources was carried out in conjunction with the study on the $(0 \overline{2} 2)$ crystal. As a part of this investigation the peak position of the $411.800 \mathrm{keV} \mathrm{Hg}^{198}$ line was determined for source position changes effected by moving the source holder carriage which is aligned normal to the focal circle. The source was an 0.001 in. Au wire. The results are presented in fig. 10 where $\delta$ represents the change in peak position. The errors for the values of $\delta$ are smaller than the circles. It is evident that $\delta$ must be very nearly linear with $\Delta R$, at least for source position changes up to an inch. However, from the properties of the focal circle one can easily show that by rotating a flat source about its vertical axis, one can correct for this apparent dependence of $\delta$ on $\Delta R$. Fig. 11 shows the results obtained with a 0.005 in. diameter wire and a $0.005 \times 0.125 \mathrm{in}$. flat source with a crystal area of $0.6 \mathrm{~cm}^{2}$. The curves have been normalized whereas the actual maximum intensity of the curve for the flat source would be about seven times the intensity for the wire source. The slight rounding near the top of the curve for the flat source is caused by the attenuation of the $411.8 \mathrm{keV}$ gamma-rays which originate near the rear of the source and must traverse the diameter of the iron cylinder holding the source foil. The leading edge of this curve is sharply defined and rises from ten per cent of its maximum intensity to the maximum intensity in $0.058 \mathrm{~s}$.d. The base widths of the two curves are equal. Increasing the crystal area a factor of seven results in a corresponding increase in the maximum intensities of each curve with no change in the fwhm. The base widths increase to 0.250 s.d. and the curve due to the flat source tends to peak and become more like the curve for the wire source. As a practical example of using a flat source, fig. 12 shows the results obtained with a $0.002 \times 0.060 \mathrm{in}$. tungsten ribbon with a strength of about $200 \mathrm{mCur}$. The alignment of the source took about five minutes. The transitions follow the decay of $\mathrm{W}^{187}$ and the quoted energies are obtained from the data for two separate measurements on each of these transitions. It is interesting to note that the fwhm, $2.75 \mathrm{keV}$, of the 618.32 $\mathrm{keV}$ line is considerably smaller than the best fwhm value, $4.3 \mathrm{keV}$, which has been reported ${ }^{5}$ ) for a 661 $\mathrm{keV}$ line using a lithium drifted germanium diode

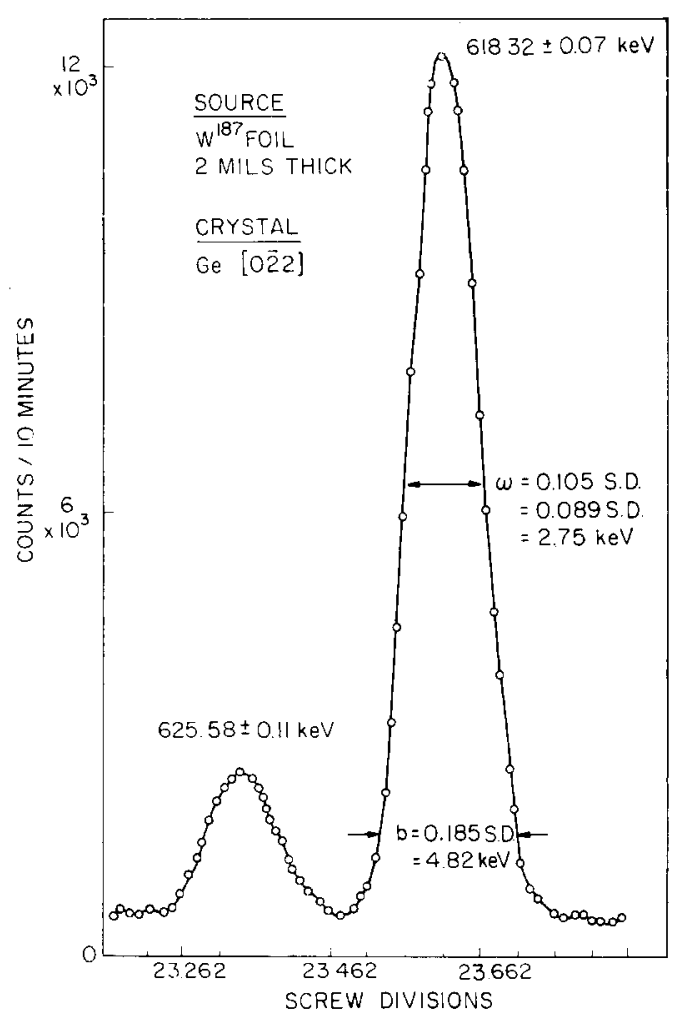

Fig. 12. The 618.32 and $625.58 \mathrm{keV}$ gamma-ray lines in $\operatorname{Re}^{187}$ observed in second order reflection from the germanium (025) planes. The source was 62 mils wide. The data have been corrected for source decay. 
TABLE 2

The parameters $\omega$ and $b$ of the diffracted peak using various crystals and source sizes. If two areas are given for a particular source and crystal combination the larger area denotes the usual configuration which is used in a measurement. The smaller area is presented to demonstrate the best possible case. Where only one crystal area is given for a particular source-crystal combination it represents the best possible case and is the value which is generally used in a measurement.

\begin{tabular}{|c|c|c|c|c|}
\hline Source & Crystal & $\begin{array}{c}\text { Crystal } \\
\text { area } \\
\left(\mathrm{cm}^{2}\right)\end{array}$ & $\begin{array}{c}\omega \\
\text { Fwhm } \\
\text { (s.d.) }\end{array}$ & $\begin{array}{c}b \\
\text { Base w } \\
\text { Fw } 10 \% \mathrm{~m} \\
\text { (s.d.) }\end{array}$ \\
\hline \multirow[t]{2}{*}{1 mil wire } & 400 & 0.2 & 0.044 & 0.084 \\
\hline & $0 \overline{2} 2$ & 0.4 & 0.041 & 0.072 \\
\hline 2 mil wire I* & 400 & 0.4 & 0.074 & 0.164 \\
\hline $2 \mathrm{mil}$ wire II & $0 \overline{2} 2$ & 0.4 & 0.080 & 0.128 \\
\hline 2 mil wire I & 112 & 4.8 & 0.135 & 0.244 \\
\hline 2 mil wire I & 112 & 7.0 & 0.200 & 0.328 \\
\hline 2 mil wire II & 310 & 20.0 & 0.217 & 0.365 \\
\hline \multirow[t]{4}{*}{5 mil wire } & 400 & 0.8 & 0.140 & 0.275 \\
\hline & 400 & 1.6 & 0.160 & 0.330 \\
\hline & $0 \overline{2} 2$ & 3.2 & 0.126 & 0.220 \\
\hline & 112 & 7.0 & 0.200 & 0.352 \\
\hline \multirow[t]{4}{*}{10 mil wire } & 400 & 0.8 & 0.250 & 0.385 \\
\hline & $0 \overline{2} 2$ & & & \\
\hline & 112 & 4.8 & 0.270 & 0.456 \\
\hline & 112 & 8.4 & 0.280 & 0.540 \\
\hline $2 \mathrm{mil} \times 50 \mathrm{mil}$ & $0 \overline{2} 2$ & 1.6 & 0.105 & 0.175 \\
\hline $3 \mathrm{mil} \times 195 \mathrm{mil}$ & $0 \overline{2} 2$ & 3.2 & 0.114 & 0.205 \\
\hline \multirow[t]{5}{*}{$5 \mathrm{mil} \times 125 \mathrm{mil}$} & 400 & 0.4 & 0.170 & 0.292 \\
\hline & 400 & 1.6 & 0.210 & 0.400 \\
\hline & $0 \overline{2} 2$ & 0.8 & 0.158 & 0.210 \\
\hline & $0 \overline{2} 2$ & 3.2 & 0.160 & 0.250 \\
\hline & 112 & 7.0 & 0.210 & 0.365 \\
\hline
\end{tabular}

* Two different two mil wires were used. Wire I seemed to be the better source but this source was subsequently damaged.

gamma-ray detector. Since the $625 \mathrm{keV}$ transition is about $1 \%$ of the total decay $^{6}$ ), the partial neutroncapture cross section contributing to this transition is about 0.4 barn. By increasing the source dimensions, the minimum partial cross-section could even be as small as 10-20 millibarn and a good determination of the energy of this transition would still be possible. In fact, the $618 \mathrm{keV}$ gamma line was measured in third order using a $0.003 \times 0.195$ in. source of $100 \mathrm{mCur}$. The fwhm of the peak was 0.114 s.d. or $1.99 \mathrm{keV}$. From these studies it was found that flat sources pose no special problems and are generally to be preferred over wire sources due to the large possible increase in source material.

\subsection{Relative PROPERTIES OF THE (310) AND (112) QUARTZ AND THE (400) AND (0 $\overline{2} 2$ ) GERMANIUM CURVED CRYSTALS}

A tabulation of the peak parameters $\omega$ and $b$ for commonly used crystal areas is presented in table 2 . Several features become evident from this table. The base width corresponding to a particular source is always larger with the $(400)$ planes than with the $(0 \overline{2} 2)$ planes although the values for $\omega$ may be nearly equal. Larger areas of the $(0 \overline{2} 2)$ crystal may be used than with the (400) crystal for similar peak parameters $\omega$ and $b$. Both germanium crystals give sharper peaks than either quartz crystal in agreement with the work of Seppi et al. ${ }^{2}$ ) on the germanium (400) planes and the quartz (310) planes.

The relative peak reflection efficiency for the 411.8 $\mathrm{keV}$ gamma-ray for different orders of reflection using the four crystals are presented in table 3 . The peak reflection efficiency corresponds to the fraction of the incident radiation which is selectively diffracted at the maximum of the line profile with corrections made for the attenuation in the crystal. The region was selected for each crystal which would result in the maximum peak counting rate thereby insuring that the best region of each crystal was used. This still does not insure that the region corresponds to a perfect or ideal crystal. Data from other crystals with the same orientations could differ from our results depending on the imperfections in the crystals. The data in the last column were obtained with the $676 \mathrm{keV}$ gamma-ray in the decay of

\section{TABLE 3}

Relative peak reflection efficiencies using the four crystals. All crystals are $2 \mathrm{~mm}$ thick. The data are obtained for a gamma-ray energy of $411.8 \mathrm{keV}$ except the last column where the energy is $676 \mathrm{keV}$. The data have been obtained for equal crystal areas and normalized so that the value corresponding to the first order reflection from the quartz (310) planes is taken to be 100 . The relative peak reflection efficiency corresponds to the fraction of the incident radiation which is selectively diffracted at the maximum of the line profile with correction made for the attenuation in the crystal.

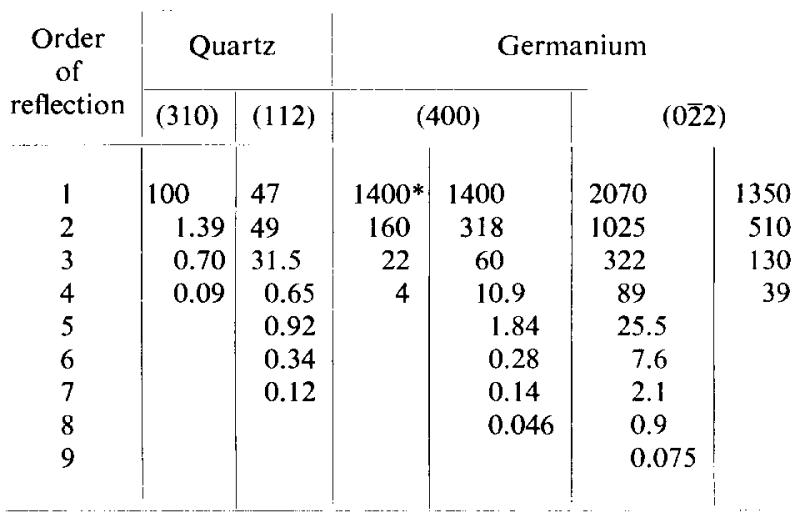

* The data in this column have been deduced from the work of Seppi et al.2) and are normalized so the first order reflection in their work and the present work are equal. 
$\mathrm{Au}^{198}$. These data have been corrected for source absorption, attenuation due to the germanium crystal, $\mathrm{NaI}(\mathrm{Tl})$ efficiency and the peak-to-total factor and compared with the $412 \mathrm{keV}$ gamma-ray using $1.1 \%$ for the partial decay of $\mathrm{Au}^{198}$ which proceeds through the $676 \mathrm{keV}$ transition ${ }^{7}$ ). Therefore, the last column presents the reflection efficiency of the germanium $(0 \overline{2} 2)$ planes for a $676 \mathrm{keV}$ gamma-ray energy where the first order reflection efficiency for the $412 \mathrm{keV}$ gamma-ray from these planes is taken to be 2070. An uncertainty of $20 \%$ is possible in the data in the last column, whereas the data in the remaining columns have a possible uncertainty of $5 \%$ except for values less than unity where the uncertainty can be as high as $20 \%$. It appears that the peak reflection efficiency at $676 \mathrm{keV}$, $\xi(676)$ decreases by a factor of $0.65 \pm 0.13$ compared to $\xi(412)$ for the first order reflection from the germanium (022) planes. In higher orders of reflection this ratio $\xi(676) / \xi(412)$ becomes smaller. With a $10 \mathrm{mil}$ $\mathrm{W}^{187}$ source and using $0.45 \pm 0.09,1.00$ and $1.25 \pm$ 0.25 for the relative intensities of the 134,479 and 686 $\mathrm{keV}$ gamma-rays it was found that $\xi(134) / \xi(479 / \xi(686)$ $=1.19 \pm 0.34 / 1.00 / 0.78 \pm 0.20$. In addition, the ratio of $\xi(134)$ for the germanium $(0 \overline{2} 2)$ planes compared with $\xi(134)$ for the quartz (310) planes was determined to be $4.85 \pm 1.0$. For the germanium $(400)$ planes the ratio $\xi(676) / \xi(412)$ was found to be $0.56 \pm 0.11$ for first order reflection. From the data of Seppi et al. ${ }^{2}$ ) this same ratio appears to be roughly 0.43 . The relative reflection efficiency values for the (400) planes deduced from the data of Seppi et al. and normalized to the first order value in the present work are given in column four. Notice that the ratios would be in better agreement with the present work if the first order value of Seppi et al. were decreased by fifty percent. It is evident from the table that the intensity generally decreases with increasing order of reflection except for the quartz (112) planes. This crystal is also unique in that the first three orders of reflection have similar reflection efficiencies. The data in table 3 are also presented in fig. 13 where the relative peak reflection efficiency has been renormalized and is plotted as a function of dispersion (or order of reflection). It is interesting that the points corresponding to the reflection of the $411.8 \mathrm{keV}$ gammaray from the planes of the two germanium crystals reported in this work are on the same straight line unless the dispersion is less than 35 s.d. From this plot one finds the relative peak reflection efficiency to be proportional to $\exp (-0.031 x)$ for $x$ greater than 35 where $x$ is the dispersion in s.d. For the $676 \mathrm{keV}$ gammaray energy the peak reflection efficiency is proportional to $\exp (-0.053 x)$ for $x$ greater than 20 . From this plot it is evident that for measurements in orders of reflection greater than one with the germanium (400) or $(0 \overline{2} 2)$ planes it is immaterial which planes are used, at least in the $400 \mathrm{keV}$ region, if one is mainly concerned with peak reflection efficiency or, equivalently, the peak counting rate. At lower energies the advantage of the germanium over the quartz is not so great and below the region of $100 \mathrm{keV}$ the quartz crystal gives the higher counting rates due to the greater attenuation of the reflected radiation in the germanium.

\section{Determination of the energies of the gamma-rays following the decay of $\mathrm{Au}^{198}$ and $\mathrm{Au}^{199}$}

The results one may obtain with the germanium crystals are demonstrated by the determination of the gamma-rays above $100 \mathrm{keV}$ which follow the decay of $\mathrm{Au}^{198}$ and the double capture isotope $\mathrm{Au}^{199}$. The energy determination by the curved-crystal method of one of these gamma-rays, the $1088 \mathrm{keV}$ transition, has never been reported. The germanium $(0 \overline{2} 2)$ planes were used in all the measurements. However, the (400)

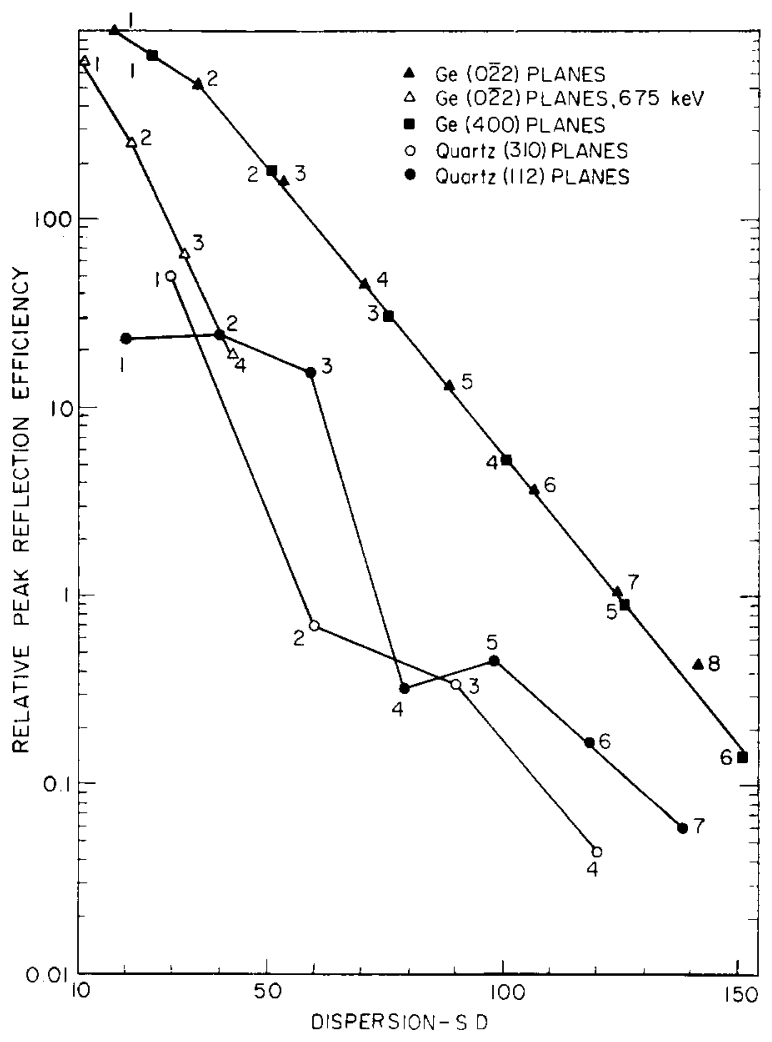

Fig. 13. Relative peak reflection efficiency of the germanium and quartz crystals vs crystal position (dispersion). The data are obtained from table 3 and are renormalized in this plot. All points represent data for a gamma-ray energy of $411.8 \mathrm{keV}$ except the open triangles ( 1 ) which represent data for an energy of $676 \mathrm{keV}$. The numbers adjacent to the points denote the order of reflection. 


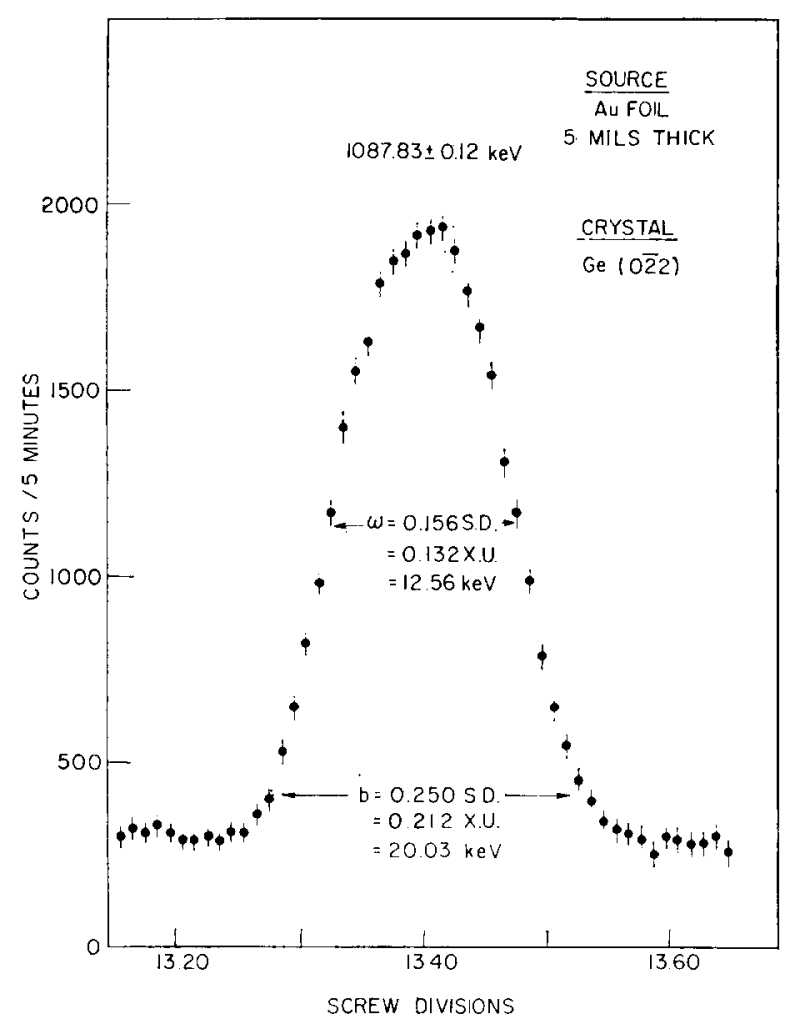

Fig. 14. The $1087.83 \mathrm{keV}$ gamma-ray line in the decay of Au198 observed in second order reflection. The source strength was approximately $3 \mathrm{Cur}$ and the source was 125 mils wide.

planes were also used in one measurement to demonstrate the agreement between energy values obtained with the two crystals. The source was a $0.005 \times 0.125$ in. gold foil, and the source strength of the $\mathrm{Au}^{198}$ was roughly 3 Cur. The results of the measurements are:

$$
\begin{array}{cc}
\mathrm{Au}^{198} & 675.90 \\
1087.83 & \pm 0.05 \mathrm{keV} \\
\mathrm{Au}^{199} & \pm 0.12 \mathrm{keV} \\
208.216 \pm 0.008 \mathrm{keV} \\
158.393 \pm 0.007 \mathrm{keV}
\end{array}
$$

The method for determining the uncertainties was discussed in section 5.2. The energy of the last gamma-ray is the result of measurements using both germanium crystals. The energy values of $158.385 \pm 0.006 \mathrm{keV}$ and $158.401 \pm 0.012 \mathrm{keV}$ were obtained using the $(0 \overline{2} 2)$ and (400) crystals, respectively. The $676 \mathrm{keV}$ energy value was obtained from four second order and four third order measurements. The $1088 \mathrm{keV}$ energy value was obtained from seven second order measurements. A typical reflection peak for this gamma-ray is shown in fig. 14. This $1088 \mathrm{keV}$ transition has been designated as the cross-over transition corresponding to the 676-
$412 \mathrm{keV}$ cascade $^{7}$ ). From the above measurements we have for the cascade sum:

$$
675.90 \pm 0.05+411.80=1087.70 \pm 0.05 \mathrm{keV} .
$$

Comparing this sum with the measured value of $1087.83 \pm 0.12 \mathrm{keV}$ for the high energy gamma-ray, it appears that this transition does indeed correspond to the cross-over with the present measurements being the best evidence to date for this designation.

The results of other authors for the measurements of the above transitions in $\mathrm{Au}^{198}$ and $\mathrm{Au}^{199}$ are presented below. The results of other authors which are presented are the energy values with the smallest uncertainties which have been published for the respective transitions. Notice that the corresponding uncertainties in the energy determinations in this work are smaller in each case.

$1087.83 \pm 0.12 \mathrm{keV}:$ Our value is in good agreement with the value of Backstrom and Bergman ${ }^{8}$ ), $1087.47 \pm$ $0.35 \mathrm{keV}$, in close agreement with the value of Elliot et al. $\left.{ }^{9}\right), 1088.9 \pm 0.9 \mathrm{keV}$, and in disagreement with the $1086.6 \pm 0.6 \mathrm{keV}$ value of Kane and Emery ${ }^{10}$ ). These three values were obtained from energy measurements on internal and external conversion electrons.

$675.90 \pm 0.05 \mathrm{keV}:$ Our value is in good agreement with the $675.75 \pm 0.25 \mathrm{keV}$ determination of Backstrom and $\operatorname{Bergman}^{8}$ ), but in disagreement with the value of $674.32 \pm 0.20 \mathrm{keV}$ which was obtained with the Argonne $7.7 \mathrm{~m}$ curved-crystal spectrometer by Hammermesh and Smithers ${ }^{11}$ ).

$208.216 \pm 0.008 \mathrm{keV}$ : Our value is in good agreement with the $208.226 \pm 0.025 \mathrm{keV}$ value of Backstrom et al. ${ }^{12}$ ), in close agreement with the value of $208.15 \pm 0.05 \mathrm{keV}$ reported by Wapstra ${ }^{13}$ ), but in disagreement with the $208.12 \pm 0.03 \mathrm{keV}$ value of Hammermesh and Smithers ${ }^{11}$ ). Our value also agrees with the less precise value of $208.17 \pm 0.12 \mathrm{keV}$ reported by Avotina and Sumboev ${ }^{14}$ ). These latter authors used the quartz (112) planes in a $2 \mathrm{~m}$ curvedcrystal spectrometer.

$158.393 \pm 0.007 \mathrm{keV}$ : Our value is in good agreement with the value of Backstrom et al. ${ }^{12}$ ) $158.396 \pm$ $0.025 \mathrm{keV}$ and of Jung and Svedberg ${ }^{15}$ ) $158.36 \pm 0.05$ $\mathrm{keV}$, but is in diagreement with the $158.335 \pm 0.015$ $\mathrm{keV}$ value of deVries and Dijkstra ${ }^{16}$ ), and the $158.33 \pm$ $0.02 \mathrm{keV}$ value of Hammermesh and Smithers ${ }^{11}$ ). The $158.27 \pm 0.35 \mathrm{keV}$ value of Avotina and Sumbaev also is in agreement with our result.

It is interesting that in every case the value determined in the present work is larger than the corresponding value obtained with the Argonne $7.7 \mathrm{~m}$ curvedcrystal spectrometer. This is further surprising in view 
of the fact that the measurement of the $412 \mathrm{keV}$ transition reported by the Argonne group was $411.79 \pm$ $0.03 \mathrm{keV}$ which is in almost exact agreement with our calibration value of $411.800 \mathrm{keV}$.

Note added in proof: Precision measurements on the transitions following the decay of $\mathrm{Au}^{198}$ and $\mathrm{Au}^{199}$ have recently been reported by G. Kaye and $R$. L. Graham, Can. J. Phys. 42 (1964) 1865. This group performed measurements on the internal conversion electron spectrum using the Chalk River 1-meter-radius $\pi \sqrt{ } 2 \beta$-ray spectrometer.

The authors wish to thank Mr. J. B. Bullock and Mr. R. Martin of the Ford Nuclear Reactor staff for many irradiations and Mr. A. B. Miller for his invaluable assistance in the maintenance of the instrument.

\section{References}

1) J. W. M. DuMond, Ann. Rev. Nucl. Sci. 8 (1958) 163.
2) E. J. Seppi, H. Henrikson, F. Boehm and J. W. M. DuMond, Nucl. Instr. and Meth. 16 (1962) 17.

3) G. Murray, R. L. Graham and J. S. Geiger, Nucl. Phys. 45 (1963) 177.

4) D. A. Lind and P. Henning, Bull. Am. Phys. Soc. 9 (1964) 549.

5) G. T. Ewan, AECL-1960;

A. J. Tave-dale, IEEE Trans. Nucl. Sci. (June, 1964) 191.

6) C. J. Gallagher, Jr., W. F. Edwards and G. Manning, Nucl. Phys. 19 (1960) 18.

7) Nuclear Data Sheets (National Academy of Sciences, National Research Council, Washington, D.C.) No. 5-2-55.

8) G. Backstrom and O. Bergman, Ark. Fys. 13 (1958) 393.

9) L. G. Elliott, M. A. Preston and J. L. Wolfson, Can. J. Phys. 32 (1954) 153.

10) W.R. Kane and G.T.Emery, Bull. Am. Phys. Soc. 3 (1958) 64.

11) B. Hammermesh and R. K. Smithers, Ann. Phys. (N.Y.) 13 (1961) 307.

12) G. Backstrom, O. Bergman and J. Burde, Nucl. Phys. 7 (1958) 263; 15 (1960) 566.

13) A. H. Wapstra, Private communication; Nuclear Data Sheets, No. 5-3-60.

14) M. R. Avotina and O. I. Sumbaev, Izvest. Akad. Nauk SSSR, Ser. Fiz. 22 (1958) 879.

15) B. Jung and J. Svedberg, Nucl. Phys. 20 (1960) 630.

16) C. de Vries and J. H. Dijkstra, Nucl. Phys. 18 (1960) 446. 